\title{
Una experiencia de asesoría en la instalación de los planes de formación ciudadana (Ley 20.911) en establecimientos de dependencia municipal de la Región Metropolitana de Chile
}

\author{
Pablo Solorzano Marchant ${ }^{1}$
}

Recibido 20 de marzo 2019 · Aceptado 03 de mayo 2019.

\begin{abstract}
Resumen:
El artículo trata de una experiencia de asesoría en la instalación de los Planes de Formación Ciudadana para el sistema escolar chileno (Ley 20.9II). El propósito del texto es evidenciar algunos nudos críticos para su implementación en el cruce entre estas nuevas exigencias y las culturas escolares. Se informa sobre una experiencia innovadora de asesoramiento a 48 escuelas de la Región Metropolitana, en la que participaron académicos de la Universidad Católica Silva Henríquez en colaboración con el Ministerio de Educación, abarcando a tres Departamentos Provinciales de Educación. La metodología de trabajo se fundamentó en una estrategia de intervención: I) trabajo interdisciplinario; 2) conocimiento del proceso de implementación del Plan de Formación, y 3) generación de capacidades para la formación ciudadana. Los resultados del primer taller masivo (2016) arrojan que los encargados de formación ciudadana entienden que la instalación del plan es "difícil, pero no imposible", depende del contexto y las interacciones, la comprensión del currículo escolar y la integración entre los documentos institucionales. Se concluye que I) existe en las culturas escolares una mirada restringida del significado de la Ley, 2) la atención a la tarea en ciudadanía es con una mirada a corto plazo, y 3 ) los actores tienden a no reconocer prácticas ad hoc a la construcción de ciudadanía.
\end{abstract}

Palabras clave: formación ciudadana, culturas escolares, construcción social, asesoramiento educativo.

An Advisory Experience on the Implementation of Citizen Training Plans (Law 20,911) in Municipal Public Schools in the Metropolitan Region of Chile

\section{Abstract:}

The article describes an advisory experience on the implementation of Citizen Training Plans for the Chilean school system (Law 20,9II). The purpose of the text is to show some key points for its implementation where these new requirements meet intersect with the school cultures. It reports on an innovative advisory experience for 48 schools in the Metropolitan Region, with the participation of faculty from the Universidad

Doctor en Educación, Universidad de Santiago de Chile. Académico, Escuela de Filosofía, Universidad Católica Silva Henríquez. Contacto: psolorzanom@ucsh.cl 
Católica Silva Henríquez in collaboration with the Ministry of Education and covering three Provincial Departments of Education. The work methodology was based on an intervention strategy: I) interdisciplinary work; 2) knowledge of the Training Plan implementation process; and 3 ) generation of skills for citizen training. The results of the first mass workshop (2016) show that the people responsible for citizen training believe that implementation of the plan is "difficult, yet not impossible," depends on the context and interactions, understanding of the school curriculum and integration among institutional documents. It is concluded that I) the student cultures have a restricted understanding of the meaning of the Law, 2) the attention given to citizen training has a short-term perspective, and 3 ) the key actors tend to not recognize ad hoc practices for citizen building.

Key words: citizen training, school cultures, social construction, educational advisory.

\section{La experiencia de asesoría en la implementación de la ley}

La Ley 20.9I I chilena es un llamado a que cada establecimiento educacional se haga cargo de la coconstrucción de la democracia desde su propia cultura escolar, como forma de hacer responsable a toda una comunidad de convivir, participar y construir desde las orientaciones que comprenden la formación ciudadana, cuestión que se expresa claramente en sus nueve objetivos. De este modo, no se reduce a una cuestión curricular, al instalar asignaturas o talleres que abarquen estos contenidos, ni menos tan solo a disponer de un conglomerado de actividades, que muchas veces termina siendo un activismo en las escuelas y que poco se relaciona con formar para la democracia. Esta Ley orienta al trabajo con toda la comunidad educativa, que se responsabiliza de construir, en una cultura democrática, participación y formación en ciudadanía.

En el contexto de un proyecto de colaboración de la Universidad Católica Silva Henríquez (UCSH) y el Ministerio de Educación de Chile (MINEDUC), fue posible advertir algunos alcances que describen y proponen una mirada al contexto y se disponen a trabajar en la construcción de un Plan de Formación Ciudadana (PFC). Estos se desprenden desde un trabajo taller realizado con equipos directivos y profesores de Enseñanza Básica y Media de la Región Metropolitana, además de las reflexiones realizadas con un equipo interdisciplinario de la UCSH, compuesto por académicos 
de Pedagogía en Historia, Pedagogía en Filosofía y de la unidad de Dirección de Formación Identitaria (DIFI).

Como antecedente respecto de la formación ciudadana, existen elementos teóricos y concretos que comprenden el nacimiento de esta ley. Primero, se publica el Informe Delors (I996), que hace hincapié en que la educación para el siglo XXI se debe constituir desde cuatro pilares. Estos indican la necesidad de abordar la ciudadanía como eje relevante para la formación. Segundo, durante estos últimos años se ha tomado conciencia de la necesidad de considerar aspectos culturales en la formación pedagógica. Nos dice Unesco, en sus directrices sobre la formación intercultural, que "la cultura, fenómeno colectivo e histórico, no puede existir sin una continua transmisión y enriquecimiento mediante la educación” (UNESCO, 2006, p. I3). De la misma forma, existen variados textos que anteceden a la formulación de la Ley desde la denominada vuelta a la democracia en Chile (Escobar, 2018)

Cuando se analizan los objetivos de la misma Ley, se propone un nuevo paradigma de cómo se deben comprender las instituciones escolares en beneficio de la construcción de una sociedad. Se trata de hablar de un ethos, una morada común; debe construirse con la participación de todos quienes en él conviven. Se debe asumir que esto coloca la gestión escolar en un lugar diferente. De algún modo, comienza a corroer un modelo de vivir y convivir en la escuela, en la que solo algunos son responsables de ella y otros obedecen. Se trata entonces de promover una mirada ética sobre las relaciones de poder y de diálogo al interior, que se faciliten los espacios de comprensión y cuidado del carácter democrático de una escuela, que la representación y la participación de los actores den verdadero cuerpo a la Ley (Arguelles, 2009)

Sin duda, los desafíos de pensar una escuela desde el contexto de la Ley y, al mismo tiempo, construir espacios que favorezcan su vida democrática, generaron más de una pregunta en el equipo de trabajo ante el proyecto de colaboración. Desde ese considerando se elaboraron tres preguntas fundamentales, transversales que, de alguna manera, aglutinaban a todas las otras inquietudes para una discusión a nivel de mesa técnica. Estas fueron: 
I. ¿En qué se fundamenta la apatía política en las formas clásicas de participación?

2. ¿Qué tipo de democracia se piensa cuando se habla de ciudadanía para el sistema escolar?

3. ¿Cuáles son los problemas asociados a la instalación de un plan de formación en el sistema escolar?

De estas preguntas, el proyecto solo se hizo cargo de la tercera, puesto que, por la premura del tiempo del proyecto y su naturaleza, las dos primeras ameritan un trabajo más extenso y difícil de responder en este tipo de experiencia, aunque sin duda es relevante de atender debido a que son transversales al ámbito de las ciencias humanas y de las ciencias sociales.

A pesar de que este texto solo se encarga de la tercera, se tuvo como supuesto de trabajo las dos anteriores. La primera es la lógica de una democracia representativa y su forma entender la participación ciudadana, que no permite que hoy cobren sentido los estímulos para actuar en los procesos del sistema electoral. Existe una apatía generalizada frente a la realidad construida por este tipo de régimen en la política, además de las estructuras y símbolos que promueve la instalación del sistema neoliberal, hacen que el sujeto padezca en su existencia de las tensiones que se generan en lo cotidiano. De esta manera, la política se padece en el ámbito más subjetivo e intersubjetivo que afecta a los modos de experiencia y de valorar la democracia (Norbert, 2002). En cuanto a la segunda, la forma de representación de la formación ciudadana está tensionada por valoraciones que hace el mismo sujeto, que lo sitúan, siguiendo a Arguelles (2009), en dos lógicas: una para una participación positiva, en la que el ciudadano se siente empoderado, participa de la construcción de las leyes y recrea un mundo político en el que el Estado no hace oídos sordos a sus demandas. Al contrario, la participación guiada y controlada por los procesos y técnicas del Estado, que permiten "escuchar" a la ciudadanía, terminan generando resignación en la acción política del ciudadano. De esta manera, habría que preguntar si la apatía política no será un síntoma de los modos de concebir la participación política por parte de los gobiernos. Pregunta relevante que no se resolverá en este artículo. 
Desde la pregunta operacional tratada $\left(\mathrm{N}^{\circ} \mathrm{I}\right)$, se diseñó un taller para relevar, desde la percepción de los equipos directivos y de los profesores participantes, las primeras nociones del PFC en su instalación y concreción, en una jornada masiva que aglutinó a los colegios de la primera cohorte (2016-2017). En el siguiente título se indicarán los hallazgos recogidos.

\section{La democracia se construye a pesar de todas sus barreras}

Para construir y desarrollar el proyecto de asesoramiento, la Universidad se propuso discutir, desde una mesa técnica interdisciplinar, los procesos y procedimientos con los que se pudiera establecer un diálogo más crítico y al mismo tiempo concluyente al problema de la formación ciudadana. En este procedimiento se descubrió la necesidad de que la elaboración del Plan recogiera, en sus instrumentos de gestión y administración escolar, tanto las acciones que se realizaban en las escuelas como las percepciones comunes respecto de su significado.

Para aglutinar las percepciones de los actores escolares se realizó el primer taller con 48 colegios. Propuso la mesa técnica investigar y conocer algunos nudos críticos que impidieran una construcción e instalación del PFC. Por tal motivo, se planteó como hipótesis de trabajo que: es posible lograr el fortalecimiento de la democracia en el sistema escolar afectando a tres ámbitos: I) las interacciones de los actores; 2) la integración de documentos institucionales, y 3) el currículo escolar.

Con el fin de captar esos nudos, se confeccionó y aplicó un instrumento de recolección de información. En este se consultó si los problemas que existían en la instalación de un PFC eran:

I) "Difíciles de cambiar, pero no imposible" o, por el contrario,

2) "Muy complejo de cambiar".

El resultado arrojó 45 respuestas en la alternativa I y, sólo 3 en la alternativa 2 . 
Los nudos críticos "Difícil de cambiar, pero no imposible", se agrupaban en dos grandes temas:

I) Precomprensiones respecto de la Ley 20.9II y

2) Estructura y funcionamiento de la cultura escolar.

En ambos se concluyó la posibilidad de intervenir sin mayores dificultades. Para mejorar la comprensión de la ley es necesario fortalecer más el propósito y corroer los prejuicios, y, a su vez, que la instalación pudiese realizarse desde los directivos y profesores para el reconocimiento de los problemas que indican en cada cultura escolar.

Respecto de las precomprensiones de la Ley, de las 45 respuestas aparecieron 60 enunciados que se agruparon en ideas, destacándose tres grandes temas (tabla I):

Tabla I. Síntesis Enunciados precompresiones de la Ley 20.9I I

PRE-COMPRENSIÓN DE LA LEY (PROFESORES Y DIRECTIVOS)

Tiempo para realización del PFC ("es más trabajo para los profesores”).

Trabajo técnico de la integralidad de la documentación de gestión ("cómo se realiza la coherencia entre los documentos institucionales y el PFC").

Proactivismo ("se puede realizar acciones, pero no significa una preocupación auténtica”).

Fuente: Ley 20.9II.

La tabla I muestra las precomprensiones respecto de la Ley y su instalación. Estos enunciados son la tendencia de las mayores reiteraciones (45) de un total de 60 participantes. La disposición con que enfrentan este nuevo acompañamiento, al incorporar una necesidad que entienden proviene desde el exterior y que agrega más tareas a las responsabilidades que ya tienen los profesores y equipos directivos, se traduce en si "fuera más trabajo para los profesores", y requieren más "tiempo y espacios reales para ejecutarlo". Del mismo modo, declaran no tener claridad acerca de cómo se realiza el trabajo a nivel de documentos institucionales, e identifican algunos, como el Proyecto Educativo Institucional (PEI) y el Proyecto Mejoramiento Educativo (PME), pero no entienden cómo se pueden acoplar con 
este nuevo PFC. También habría una serie de acciones que se realizan en las instituciones escolares, pero tienden a incorporar a cualquier plan (por ejemplo, convivencia escolar, inclusión, gestión pedagógica, etc.), aunque no fueran diseñados para esos fines. En este sentido, se dice de las acciones que se realizan no son una "preocupación auténtica”.

En cuanto a la estructura y funcionamiento de la cultura escolar, algunos enunciados con los que era posible cambiar la disposición para la instalación de un PFC son (tabla 2):

Tabla 2. Enunciados de las condiciones de la cultura escolar para la instalación del PFC

CONDICIONES DE LA ESTRUCTURA Y FUNCIONAMIENTO DE LA CULTURA ESCOLAR PARA LA FC (PROFESORES Y DIRECTIVOS)

Fuerza en el liderazgo directivo ("la potencia de la instalación de un plan de formación ciudadana depende del liderazgo").

Reinterpretación del valor de los actores escolares ("cambio en los modos de concebir la participación de los actores").

Mejoramiento de los canales de participación ("falta de consolidación de los canales de participación al interior de los establecimientos educacionales").

Mejoramiento del recurso en la escuela ("necesidad de incorporar recursos económicos y humanos para mayor impacto").

Fuente: elaboración propia adaptado, 2018.

En la tabla 2 se muestra la importancia del liderazgo para ejecutar este tipo de plan y la comunicación fluida que debiera generarse entre los actores educativos. Esta forma alude a la necesidad de entender los modos de participación de manera distinta, en los que exista participación real. También se indica la necesidad de que los establecimientos educacionales incorporen mayores recursos económicos y humanos para un mayor impacto del Plan en la cultura escolar.

Ciertamente, frente a estos dos nudos críticos, la incidencia en la precomprensión de la Ley se presentó como el camino corto para corroer los errores en su comprensión para la instalación del PFC. El camino largo, en cambio, debía afectar a la cultura escolar de 
cara al cambio mayor en el establecimiento. En este sentido, a medida que avanzaba el acompañamiento, se visibilizó que, para concretar una forma de participación en la cultura escolar, se debía aclarar su significado y, además, construir nociones que permitieran nuevamente mirar la escuela desde las acciones que ya se realizaban y que tributaban a este tipo de Plan, y no tensionarla.

Luego de la conclusión, la mesa técnica propuso nuevos focos de intervención. Se trata ahora de velar por la relación entre PFC y las nociones que se tengan de la cultura y el clima escolar. Esto para corregir los errores de comprensión respecto de la Ley 20.9 I I y para la construcción del mismo Plan. Algunas de estas convicciones fueron:

I. La instalación de un Plan de Formación no consiste en dotar de más responsabilidad a las escuelas y liceos, restando tiempo de su trabajo y/o quitando el espacio a otras actividades para generar mayor participación de profesores. Muchos establecimientos concebían la Ley como un documento más y un añadido a los ya existentes. Frente a esto, se hizo necesario replantear la planificación de actividades, que habitualmente no son percibidas como fundamentales en los procesos de formación y que requieren ser visibles en toda la comunidad escolar, como por ejemplo gobiernos escolares, actos escolares, desarrollo de valores sellos, entre otros. Así se incorpora lo que es parte de la cultura escolar o de la comunidad, y no se inventan o crean acciones o actividades que distorsionan a la misma Ley y a la vida u organización de la escuela.

2. Un PFC no se despliega tan solo en una asignatura sino en todas las del currículo escolar. Esto significa que los profesores deben adquirir conciencia y poner énfasis en aquellos contenidos disciplinarios y actitudes que tributan a un Plan mayor, a una visión de escuela, sociedad e interacciones humanas, que pongan en el centro la dignidad humana en la construcción de una sociedad desde la experiencia del diálogo reflexivo. Es por esto que no solo el profesor de Historia o el profesor de Filosofía serían los llamados a cuidar este espacio en la formación curricular. Incluso existen algunos talleres en colegios que tratan estas temáticas y entienden la necesidad de convertirse en agentes responsables de una tarea 
mayor; por ejemplo, en uno de estos colegios acompañados existen talleres de formación ciudadana dictados por profesores de distintas asignaturas — de química, educación física, artes, entre otras-, y fue una muy buena experiencia, tanto para los mismos profesores como para sus estudiantes. También es importante que se adquiera conciencia de que las acciones, discursos y formas de comunicación entregadas por un profesor son siempre formas de comprensión y construcción del ser ciudadano. Este es otro motivo por el que los docentes, en sus prácticas pedagógicas, deben procurar proveer siempre una formación democrática y participativa.

2. Es posible integrar los documentos institucionales a la luz de un PFC que otorgue a la institución una finalidad mayor. La relación entre los documentos institucionales con el Plan permitirá una mayor comprensión e intención de lo que se pretende desde la misión y visión institucional. De este modo, es toda una comunidad la que se moviliza en pos de la formación ciudadana y no solo un aspecto o área del establecimiento. Por otro lado, existe una comprensión en los establecimientos educacionales de que el PFC es otro Plan más, que se anexa a los existentes. Pero no se entiende que puede ser por su misma conceptualización y su expresión paradigmática que la orientación de la Ley es mayor, o que se trata de un paraguas conceptual mayor en el que es posible adherir los otros, dándoles consistencia a los documentos en general y otorgando vitalidad a los mismos sellos de los establecimientos educacionales. Esta claridad respecto a "re-mirar" el sentido de misión social por medio de la escuela, permite que los mismos documentos tengan un impacto relevante en la motivación para los actores escolares.

Tal como lo muestra la tabla 3, la integración de documentos institucionales es posible. Si se analiza los componentes de los documentos institucionales, casi todos poseen una misma conceptualización, o se desprenden unos de otros en términos de logicidad y operacionalidad. A pesar de esto, los profesores y equipos directivos no perciben su integración, y, específicamente, cómo incorporar el PFC. En este sentido, queda mucho trabajo técnico por aprender y por realizar. Es desafío de la gestión escolar prever esta situación, con el fin de generar comprensiones y acciones 
coherentes y no un exceso de acciones o documentos que, a fin de cuentas, caen en el sin sentido.

Tabla 3. Componentes de los documentos institucionales integrados (PEI, PME; PFC)

\begin{tabular}{|c|c|c|}
\hline \multicolumn{2}{|c|}{ COMPONENTES DOCUMENTOS INSTITUCIONALES } \\
\hline PEI & PME & PFC \\
\hline Descripción & Descripción & Descripción \\
\hline Misión & Misión & Diagnóstico \\
\hline Visión & Visión & Sellos \\
\hline $\begin{array}{c}\text { Objetivos Establecimientos } \\
\text { Educacionales }\end{array}$ & Objetivos Operacionales & $\begin{array}{c}\text { Objetivos PFC integrados con los } \\
\text { anteriores }\end{array}$ \\
\hline Sellos & Sellos & $\begin{array}{c}\text { Ley de Formación Ciudadana } \\
\text { (20.9II) }\end{array}$ \\
\hline Modelo Educativo & $\begin{array}{c}\text { Ámbitos de desarrollo } \\
\text { (Leyes) }\end{array}$ & $\begin{array}{c}\text { Planificación ajustada a objetivos } \\
\text { de la Ley }\end{array}$ \\
\hline Estructura Organizacional & Planificación Estratégica & Acciones \\
\hline Funcionamiento & Acciones & \\
\hline
\end{tabular}

A pesar de lo anterior, como se puede apreciar en la tabla 3, la integración de los documentos por sus componentes no debiera ser una dificultad. Se puede atribuir, al contrario, que una alta complejidad de esta acción radica en su unión conceptual o que los mismos actores se pongan de acuerdo respecto de cómo entienden la participación para incluir las ideas en aquella integración. En el plano de la gestión educativa, una dificultad mayor radica en buscar esas instancias de diálogo en vistas a la creación de un ideal compartido que haya nacido en un trabajo conjunto, para dotar de una mirada que propenda a la construcción de democracia. En este sentido, se trata de generar espacios de participación, que traten acerca de temas que les incumban a los mismos actores, que puedan consensuar, a partir de ideas, qué se espera en un futuro y cuál es el horizonte compartido del mismo establecimiento educacional para organizar la tarea.

3. Las acciones incluidas en un Plan de Formación Ciudadana no significa hacer por hacer. Es retomar el espíritu de la Ley y encausarla en un mundo adulto en diálogo con el mundo juvenil. La misión es construir una casa común, de responsabilidad, para que tenga sentido la formación. En un mundo de superposición de distintos relatos en un mismo sujeto, proyectar una sociedad por construir es asumir e imaginar qué se espera. Pero, a pesar de aquello, que lo 
que se proyecta pueda tener una mirada constructiva de sociedad. Sin duda, son variados los estímulos que afectan al sujeto común en la interacción cotidiana y que obstaculizan su adecuada realización. A pesar de este gran inconveniente, se hace necesario no claudicar en el esfuerzo.

Considerando lo anterior y a pesar de que ya se vislumbran algunas orientaciones y pormenores, quedan más preguntas y desafíos que se desprenden desde esta experiencia de asesoría a los establecimientos educacionales. En lo que sigue se precisarán algunas conclusiones y algunos desafíos.

\section{Algunas conclusiones y desafíos}

\section{La construcción de ciudadanía se dificulta o potencia por las culturas escolares}

Es sabido que la Educación para el siglo XXI (Delors, 1996) indica claramente que son cuatro los pilares que se deben fortalecer en los sistemas educativos. "Aprender a ser" y "aprender a convivir juntos" son los que menos énfasis tienen en el sistema escolar, que estimula preferentemente el logro de aprendizajes principalmente académicos de tipo cognitivo. Si bien estos incluyen un "saber actuar", son las normas culturales, las estructuras sociales, el orden político y sus impactos y significados los que operan en la conformación de identidades de los actores escolares. Estas visiones están en juego en la integración o dispersión. "Actuar" se relaciona también con la historia de un pueblo, de una nación, de una zona geográfica, y se replica en continuidad por medio del traspaso de formas de cómo interpretar la sociedad y valorarla en espacios de aprendizajes compartidos. En este sentido, el interactuar y construir no es tan solo un tema educativo, sino de asumir los aspectos culturales y recoger las tensiones sociales producto de sus estructuras y significados, lo que genera modos de interpretar la realidad o de asumir como propios los discursos. La tarea por hacer es mejorar las habilidades en pensamiento crítico, de modo que el actuar mismo esté compuesto por aquellas capacidades comprensivas de la realidad que permitan asumir responsablemente 
las decisiones en el juego comunicativo. Bajo esta visión, pensar un futuro ciudadano significa también proyectar un mañana desde un ahora, que no evite el conflicto, sino que fortalezca las capacidades de dialogar, de comprometerse y de construir una mirada común. Habría que evaluar hoy qué tan preparadas están en sus creencias las instituciones escolares para permitir el cuestionamiento, que se "miren" sus prácticas, que distribuyan parte del poder para construir democracia en un marco de participación con sentido. Ciertamente es una tarea titánica, porque implica no solo hacerse cargo de la historia de nuestro país, sino además cuestionar cómo se instalaron aquellos discursos constitutivos del sistema educacional, en sus teorías, prácticas, tecnologías, así como también "re-pensar" cómo se llegó a comprender que aquellas creencias son naturales.

\section{Tendencia a no entender la integralidad de un Plan de Formación Ciudadana}

Ya casi tres décadas han pasado desde que Edgar Morín (Morín, I990) planteó una crítica a los modos de pensamiento para la producción de conocimiento, indicando que el pensamiento simple desplegado por el método científico genera impacto en los sujetos, cortándoles la carne en la vida cotidiana. La ceguera del pensamiento simple no permite ver el todo y asume que las partes pueden ser generalizadas, opacando la urdimbre existente en todo fenómeno. Traer uno de sus postulados nos permite entender la falta de integralidad que asumen también las instituciones escolares, en tanto su modo de utilizar los dispositivos constituyentes e instituyentes para su gestión educativa - planificaciones, normativas, metodologías, entre otras - se aplican y se construyen de manera parcelada, obnubilando la posibilidad de encausar en un sentido participativo. Se realizan técnicamente y en la ejecución se pierde la narrativa que da sentido. Para incorporar la integralidad de las acciones explicitadas en los mismos instrumentos es necesario rescatar la tradición, construir los recuerdos y actualizar la institución en su identidad. Desde esta perspectiva, no es solo el relato que orienta, sino la descripción de cómo las estructuras se han significado en un presente, cómo han mediado las técnicas y 
tecnologías para la producción de conocimiento, cómo las prácticas se han venido realizando y la importancia de volver a comprenderlas para proyectar la narrativa actualizada, para proyectarla en un futuro. Aquel horizonte es el desafío, llenar de sentido a la construcción de futuro en clave de formación ciudadana.

\section{La mirada a corto plazo de la construcción de un Plan de Formación Ciudadana}

Uno de los grandes problemas para el hombre en la historia occidental ha sido poder dar una definición del tiempo. No solo nos direcciona y controla convencionalmente en las acciones cotidianas, sino que en tanto existe mayor cantidad de tareas expresadas en un corto tiempo, mayor es aún la falta de eficiencia respecto de una visión expresada en un futuro. También, es un problema de lenguaje: no es lo mismo decir que existe un pasado, presente y futuro, en continuo, en progreso, que decir está "un futuro por venir" y "un pasado que se actualiza en el presente"; esto habla de que el tiempo se vive de diferentes modos, $y$, en relación con la tarea, no necesariamente lo que se espera se consigue en un tiempo cronológico. Traer esta perspectiva respecto del modo cómo se calibra el tiempo en una proyección de sentido para los establecimientos educacionales, permite entender que un Plan de Formación Ciudadana que se origine bajo la fuerza de las indicaciones de la Ley no es una tarea tan solo para ser planificada en un corto plazo. De la misma manera que el "efecto Pigmalión", se trata de proyectar a I0, 20 o 30 años la sociedad que queremos y aquella que nos dé sentido. Por lo mismo, imaginar, construir narrativa para nuestra identidad social, implica necesariamente reconocer lo que somos y ver un horizonte en clave de formación ciudadana. Aunque una advertencia antes de emprender el viaje: el sentido se parece más a un espejismo de un oasis en el desierto que a un horizonte de posibilidad; a medida que avanzamos se nos va diseminando y, por tanto, lo importante no es el final sino el recorrido. Por lo mismo, hay que reconstruir el día a día, sabiendo de los problemas de la instalación de la Ley para que, de alguna manera, se esté atento a mejorar y a percatarnos cuando los problemas ya sean otros. 


\section{Referencias bibliográficas}

Delors, J. (1996). La Educación Encierra un Tesoro. Madrid: Siglo XXI.

UNESCO. (2006). Directrices de la UNESCO sobre Formación Intercultural. París: Organización de Naciones Unidas para la Educación, Ciencia y Tecnología.

Escobar, R. (2018). Escuela y democracia. Experiencias en formación ciudadana en establecimientos educacionales de la región metropolitana. Saberes Educativos, (2), 57-75.

Arguelles, P. (2009). Participación ciudadana en el diseño de la política educativa: una mirada a la contrucción de ciudadanía en el diálogo ciudadano. Foro Educacional, I05-I24.

Norbert, L. (2002). Las sombras del mañana. La dimensión subjetiva de la política. Santiago de Chile: LOM.

Morín, E. (1990). Introducción al Pensamiento Complejo. Madrid: Gedisa.

Ministerio de Educación. (2016). Ley 20.911. Santiago deChile: Congreso de Chile. 